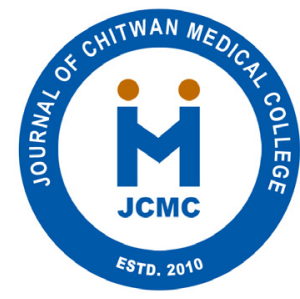

\title{
INFLAMMATION AND OXIDATIVE STRESS VIA PERSISTENT HYPERGLYCEMIA IN PROGRESSION OF DIABETIC NEPHROPATHY IN TYPE 2 DIABETES MELLITUS
}

\author{
D Kafle ${ }^{1}$, N Islam ${ }^{2}$, B Aryal ${ }^{3}$, P Adhikary ${ }^{3}$ and N Singh ${ }^{4 *}$ \\ ${ }^{1}$ Department of Biochemistry, Chitwan Medical college, Bharatpur-10, Chitwan, Nepal. \\ ${ }^{2}$ Department of Physiology, Chitwan Medical college, Bharatpur-10, Chitwan, Nepal. \\ ${ }^{3}$ Department of Clinical Pharmacology, Chitwan Medical college, Bharatpur-10, Chitwan, Nepal. \\ ${ }^{4}$ Department of Biochemistry, GR Medical College, Gwalior, India. \\ *Correspondence to : Prof Dr Neelina Singh, Department of Biochemistry, GR Medical College, Gwalior, India. Email: neelima48@rediffmail.com
}

\begin{abstract}
Diabetic nephropathy is a major microvascular complication of diabetes, representing the leading cause of end stage renal disease in the world, and a major cause of morbidity and mortality in type 2 diabetic subjects. In the kidney, a number of pathways that generate reactive oxygen species (ROS) such as glycolysis, specific defects in the polyol pathway, uncoupling of nitric oxide synthase, xanthine oxidase, NAD (P) H oxidase, and advanced glycation have been identified as potentially major contributors to the pathogenesis of diabetic kidney disease. Changes in oxidative stress biomarkers, including superoxide dismutase, catalase, glutathione reductase, glutathione peroxidase, glutathione levels, vitamins, lipid peroxidation, nitrite concentration, nonenzymatic glycosylated proteins have been associated with diabetic nephropathy due to oxidative stress induced hyperglycemia. Oxidative stress in diabetes is responsible for endothelial dysfunction releasing inflammatory markers cytokines from the damaged renal tissue. Hyperglycemia induces intracellular reactive oxygen species in mesangial and tubular epithelial cells which induces cytokines, IL-6 and TNF- $\alpha$ production in glomerular mesangial and tubular epithelial cells in diabetic kidney. Antioxidants inhibit high glucose induced transforming growth factors and extra cellular matrix expression in glomerular mesangial and tubular epithelial cells, which ameliorate features of diabetic nephropathy, suggesting that oxidative stress plays an important role in diabetic renal injury causing diabetic nephropathy.
\end{abstract}

Key Words: Inflammatory markers, oxidative stress, diabetic nephropathy \& endothelial dysfunction

\section{INTRODUCTION}

Diabetic nephropathy is the most common cause of microvascular chronic complication of type 2 diabetes mellitus which is associated with considerable morbidity and mortality, finally leading to end-stage renal disease. ${ }^{1}$ Diabetic nephropathy is a progressive disease that takes several years to develop. Glomerular hyperfiltration and increased excretion of urinary albumin (microalbuminuria) are early manifestations of diabetic nephropathy. It also involves various functional clinical abnormalities of the kidney such as elevated creatinine, urea, albuminuria, decline glomerular filtration rate, elevated arterial blood pressure, and fluid retention. ${ }^{2,3}$ Inflammation appears to be a major mechanism responsible for vascular damage leading to the clinically well-recognized complications of diabetes. Inflammation within the kidneys are due to metabolic and hemodynamic factors with locally released cytokines, such as TNF- $\alpha$, IL- 6 forming renal lesion through several mechanisms, including direct cellular injury, alteration of the glomerular protein permeability barrier, and development of intrarenal inflammatory damage all leads to pathogenic mechanism of diabetic nephropathy. ${ }^{4}$ Activation of growth factors and adhesion molecules may promote the movement of inflammatory cells into the renal microvasculature, predisposing to the development of diabetic nephropathy. ${ }^{5}$ The pathogenesis of type 2 diabetic nephropathy is characterized by a combination of insulin resistance at the level of skeletal muscle, fat, and liver, and failure of pancreatic $\beta$-cells to compensate for the enhanced insulin demand which leads to raised IL-6 levels in diabetics without glycemic control revealed the presence of inflammation causing patients more prone to neural and renal damage as well. ${ }^{6}$ Endothelial, mesangial, glomerular and tubular epithelial cells can synthesize pro-inflammatory cytokines which increases vascular endothelial permeability, proliferation of mesangial cells and matrix synthesis, as well as intraglomerular microcirculatory abnormalities. 7 Interleukin-6 affects extracellular matrix dynamics at mesangial and podocyte levels, stimulates mesangial cell proliferation, increases fibronectin expression and enhances endothelial permeability. ${ }^{8}$ Finally, in recent years, most of the attention has been focused on the implications of cytokines in the setting of diabetic nephropathy. 
INFLAMMATION CAUSING ENDOTHELIAL DYSFUNCTION IN DIABETIC NEPHROPATHY

Hyperglycemia in type 2 diabetes induces endothelial damage causing DN through four pathways: (a) increased polyol pathway flux; (b) increased advanced glycation end-product formation; (c) activation of protein kinase $\mathrm{C}$; and (d) increased hexosamine pathway flux. ${ }^{9}$

The role of inflammation in progressive tubulointerstitial renal injury leading DN correlates with a progressive decline in kidney function resulting kidney failure. ${ }^{10,11}$ Recruitment of macrophages into the glomerulus appears cells to generate reactive oxygen species with production of superoxides by the mitochondria which are considered as a common link between metabolic pathways,${ }^{12,13}$ inflammatory cytokines and proteases that induces the glomerular damage. Inflammatory cytokines, such as TNF- $\alpha$ and other mediators may stimulate glomerular cells to enhance production or reduce degradation of matrix proteins. ${ }^{14}$ Macrophage accumulates in the tubulointerstitium of animals with diabetes which leads to tubular damage increasing numbers of myofibroblasts resulting fibrosis causing endothelial dysfunction. ${ }^{15}$ Alterations in endothelial dysfunction causes elevated expression and plasma levels of vasoconstrictors such as angiotensin II and endothelin-1, increased expression of adhesion molecules associated with enhanced adhesion of platelets and monocytes to vascular endothelium, along with impairment of NO release and reduced NO responsiveness causing renal injury. ${ }^{16}$ Endothelial expression of adhesion molecules is enhanced by exposure to dyslipidemia, hypertensive plasma vasoconstrictor concentrations and elevated adipose derived pro-inflammatory cytokine levels. All these physiological changes promotes leukocyte adhesion and vascular extravasation activating nuclear factor-Kappa binding factor causing expression of m-RNA inflammatory genes via transcription increasing the inflammatory cytokines (IL-6 and TNF- $\alpha$ ), causing renal injury affecting endothelium in type 2 diabetes. ${ }^{17}$

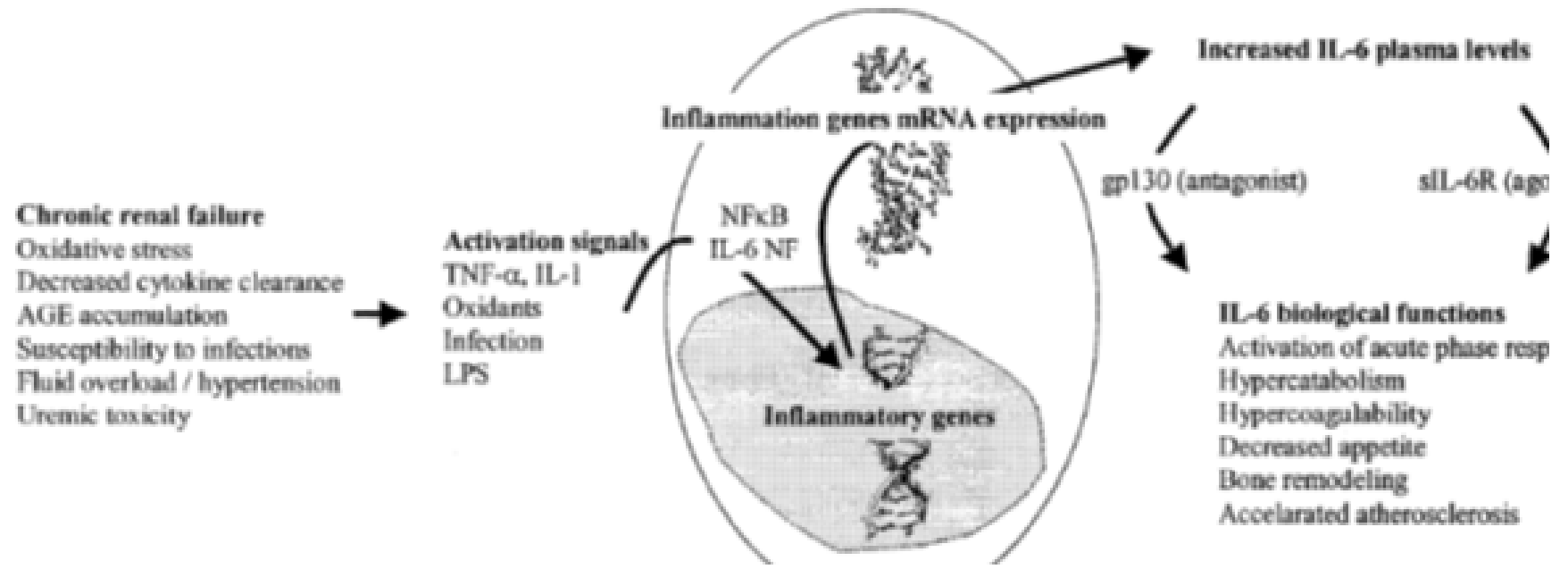

Figure 1: Schematic view of IL-6 stimulation in chronic renal failure, from causative factors related to uraemia, activation of nuclear factors that trigger the transcription of inflammatory related proteins generating increased plasma IL-6, which will perform its biological functions according to the regulation of soluble receptors. AGE, advanced glycation end-products; LPS, lipopolysaccharide; NFkB, nuclear factor kappa B; IL-6 NF, interleukin-6 nuclear factor.

\section{OXIDATIVE STRESS LINKING INFLAMMATION IN DIABETIC NEPHROPATHY}

The ability of reactive oxygen species and reactive nitrogen species to be reduced or removed by endogenous antioxidant enzymes is a key step in the limitation of tissue injury. During both acute and chronic renal injury, many intracellular and extracellular antioxidant systems become depleted, resulting in increased oxidative stress within the tissue. Redox reactions of thiol proteins are thought to be major mechanisms due to increased levels of systemic oxidative stress. The plasma protein reduced thiols (located primarily on the albumin molecule) are depleted in patients with acute kidney injury and are, thus, not able to participate in antioxidant defense. ${ }^{16}$ The protective reduced thiols are depleted in kidney injury; oxidized thiols that include homocysteine and cysteine accumulate and may have toxic effects on the endothelium. ${ }^{18}$

Protein thiols are major sources of antioxidant defense and they participate in redox signaling. (A) Cysteine residues are particularly susceptible to oxidative modification and can lead to inactivation of enzymes or binding sites. Glutathione and other thiols can reversibly oxidize cysteine residues regulating signal transduction and cellular metabolism. (B) Peroxiredoxin and other thioredoxin molecules can also reverse mild oxidative modifications of proteins. GSH, glutathione; ROS, reactive oxygen species; RNS, reactive nitrogen species; Prx, peroxiredoxin.

Macrophages can produce a broad spectrum of potential inducers of renal injury increasing the expression of IL-6, TNF- $\alpha$, upregulation in the injured kidney. 19,20 Podocytes are also considered as the major source of other inflammatory markers (IL-1 $\alpha$ and IL-1 $\beta$ ), at high glucose levels, producing monocytes chemoattractant protein-1. These molecules promote inflammation and induce further expression of macrophage 

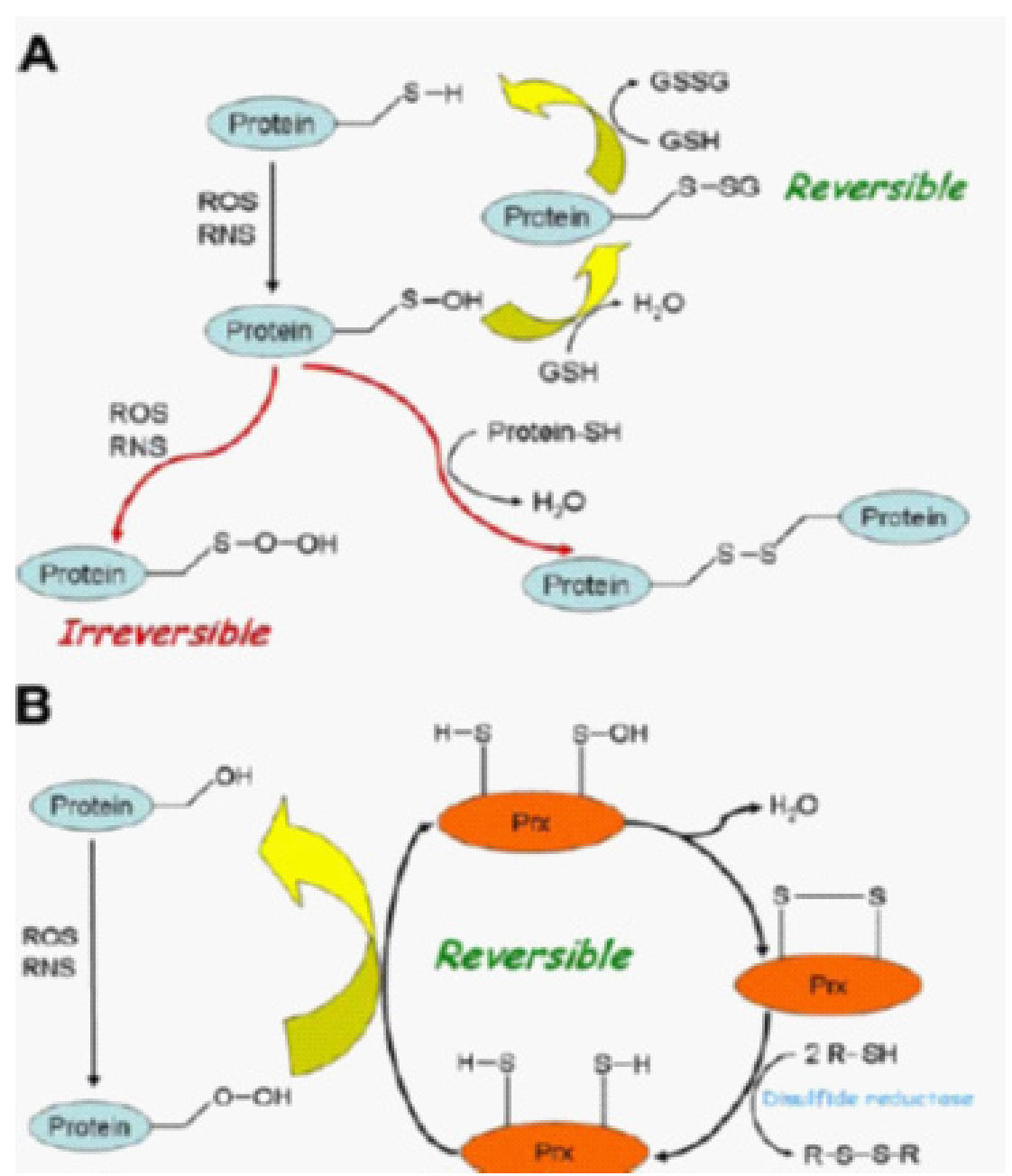

Figure 2: Modification of Proton via ROS and RNS

colony-stimulating factor and intracellular adhesion molecule -1 in renal cells. ${ }^{21}$ Once activated, macrophages release nitric oxide, reactive oxygen species, IL- 6 , TNF- $\alpha$, complement factors, and metalloproteinases, all of which promote renal injury. Moreover, activated macrophages. secrete factors such as platelet derived growth factor that promote fibroblast proliferation. ${ }^{22}$ It is interesting that renal fibrosis as measured by transforming growth factor- $\beta 1$ expression, collagen IV, and interstitial $\alpha$-smooth muscle actin was dramatically reduced in intracellular adhesion molecule -1 deficient mice, which is a key event in disease progression. The mice that are deficient in intracellular adhesion molecule -1, defective in macrophage homing into renal tissues have shown significant reduction in renal injury. ${ }^{23}$ During inflammation, neutrophil induces endothelial dysfunction by production of elevated levels of reactive oxygen species and release of cytotoxic proteinases via NADPH oxidase, a membrane-associated enzyme that generates a family of reactive oxygen species. ${ }^{24}$ Upon neutrophil activation, specific granules that contain microbial peptides, proteins, and proteolytic enzymes are released. ${ }^{25}$ It has been reported that neutrophils from patients with diabetes show increased release of oxygen radicals, such as superoxide anion and hydrogen peroxide that might damage endothelial cells and accelerate the progression of diabetic nephropathy. ${ }^{26,27}$ There is an increasing body of evidence suggesting that neutrophils from patients with diabetes display an activated phenotype, which is reflected by elevated spontaneous adhesion molecule, TNF- $\alpha$ stimulated production of superoxide free radical generating oxidative stress along with decreasing antioxidant enzymes such as glutathione peroxidase and glutathione reductase in patients with type 2 diabetes. ${ }^{28}$

\section{CONCLUSION}

Inflammatory markers, the major mediator of the acute-phase response, along with antioxidant enzyme levels are decreased in the plasma of diabetic nephropathy patients due to hyperglycemia induced oxidative stress in type 2 diabetes mellitus. Different factors prevalent in patients with diabetic nephropathy (ESRD), such as oxidative stress, hypertension, adiposity, insulin resistance, fluid overload and persistent infections which might be associated with elevated inflammatory markers (IL-6,TNF- $\alpha$ ). In addition, reduced renal function, directly or indirectly, seems to be closely related to IL-6 elevation as well as genetic expressions of various cytokines can cause endothelial dysfunction via oxidative stress might be a probable cause of diabetic nephropathy in type 2 diabetic subjects. The renal risk score for diabetic nephropathy emphasizes the importance of the identification of levels of inflammatory markers, antioxidant levels, albuminuria, hypoalbuminemia as well as increased serum creatinine, and decreased hemoglobin levels to predict the development of ESRD in patients with type 2 diabetes mellitus. 


\section{REFERENCES}

1. Raine AEG. Epidemiology, development and treatment of end-stage renal failure in type 2 (non-insulin-dependent) diabetic patients in Europe. Diabetologia 1993;36:10991104.

2. Wolf G, Ziyadeh FN. Cellular and molecular mechanisms of proteinuria in diabetic nephropathy. Nephron Physiol 2007;106:26-31.

3. Balakumar P, Chakkarwar VA, Kishan P. Vascular endothelial dysfunction: a tug of war in diabetic nephropathy?Biomed Pharmacother 2009;63:171-179.

4. Carmen M, Navarro J F. From the Nephrology Service and Research Unit. Hospital Universitario Nuestra Sen ora de Candelaria, Santa Cruz de Tenerife, Tenerife, Spain (2004).

5. Mavridis G, Souliou E, Diza Symeonidis E. Inflammatory cytokines in insulin-treated patients with type II diabetes (2007).

6. Joshi SV, Tambwekar SR, Khadalia K, Dhar HL. Role of Inflammatory Marker Interleukin 6 (IL-6) and Insulin in Diabetes and Diabetic Neuropathy. Bombay Hospital Journal (2008).

7. Royall J, Berkow RL, Beckman JS, Cunningham MK, Matalon S, Freeman BA. Tumor necrosis factor and interleukin 1 increase vascular endothelial permeability. American Journal of Physiology 1989;257: L339-L410.

8. Hirano T, Akira S, Taga T, Kishimoto T. Biological and clinical aspect of interleukin 6. Immunol Today 1990;11:443449.

9. Hovind P, Tarnow L, Rossing P. Predictors for the development of microalbuminuria and macroalbuminuria in patients with type 1 diabetes: inception cohort study. Br Med J 2004;328:1105.

10. Schmid H, Boucherot A, Yasuda Y. Modular activation of nuclear factor-kappaB transcriptional programs in human diabetic nephropathy. Diabetes 2006;55:2993-3003.

11. Navarro JF, Milena FJ, Mora C, Leon C, Garcia J. Renal Pro-inflammatory cytokine gene expression in diabetic nephropathy: effect of angiotensin-converting enzyme inhibition and pentoxifylline administration. Am J Nephrol 2006;26:562-70.

12. Seaquist ER, Goetz FC, Rich S, Barbosa J. Familial clustering of diabetic kidney disease: Evidence of genetic susceptibility to diabetic nephropathy. N Engl J Med 1989;320: 1161-1165.

13. Pettitt DJ, Saad MF, Bennett PH, Nelson RG, Knowler WC. Familial predisposition to renal disease in two generations of Pima Indians with Type 2 (non-insulin-dependent) diabetes mellitus. Diabetologia 1990;33:438-443.

14. Navarro JF, Milena FJ, Mora C, Leon C and Garcia J. Renal Proinflammatory cytokine gene expression in diabetic nephropathy: effect of angiotensin-converting enzyme inhibition and pentoxifylline administration. Am J Nephrol 2006;26:562-70.
15. Tesch GH. Role of macrophages in complications of type 2 diabetes. Clin Exp Pharmacol Physiol 2007;34:1016-9.

16. Sowers JR, Epstein M. Diabetes mellitus and associated hypertension, vascular disease, and nephropathy. Hypertension 1995;26: 869-79.

17. Prior JO, Quinones MJ, Hernandez-Pampaloni M. Coronary circulatory dysfunction in insulin resistance, impaired glucose tolerance, and type 2 diabetes mellitus., Circulation 2005;111:2291-8.

18. Scholze A, Rinder C, Beige J, Riezler R, Zidek W, Tepel M. Acetylcysteine reduces plasma homocysteine concentration and improves pulse pressure and endothelial function in patients with end-stage renal failure. Circulation 2004;109:369-374.

19. Chow F, Ozols E, Nikolic-Paterson DJ, Atkins RC, Tesch GH. Macrophages in mouse type 2 diabetic nephropathy:Correlation with diabetic state and progressive renal injury. Kidney Int 2004;65: 116-128.

20. Stehouwer CD. Endothelial dysfunction in diabetic nephropathy: State of the art and potential significance for non-diabetic renal disease. Nephrol Dial Transplant 2004; 19: 778- 781 .

21. Frank J, Engler-Blum G, Rodemann HP, Muller GA. Human renal tubular cells as a cytokine source: PDGF-B,GMCSF and IL-6 mRNA expression in vitro. Exp Nephrol 1993;1: 26-35.

22. Chow FY, Nikolic-Paterson DJ, Atkins RC, Tesch GH. Macrophages in streptozotocin-induced diabetic nephropathy:Potential role in renal fibrosis. Nephrol Dial Transplant 2004;19:2987-2996.

23. Chow FY, Nikolic-Paterson DJ, Ozols E, Atkins RC, Tesch GH. Intercellular adhesion molecule-1 deficiency is protectiveagainst nephropathy in type 2 diabetic $\mathrm{db} / \mathrm{db}$ mice. $\mathrm{J}$ Am Soc Nephrol 2005;16: 1711-1722.

24. Lambeth JD. NOX enzymes and the biology of reactive oxygen. Nat Rev Immunol 2004;4: 181-189.

25. Burg ND, Pillinger MH. The neutrophil: Function and regulation in innate and humoral immunity. Clin Immuno 2001;199: 7-17.

26. Watanabe A, Tomino Y, Yokoyama K, Koide H. Production of hydrogen peroxide by neutrophilic polymorphonuclearleukocytes in patients with diabetic nephropathy.J Clin Lab Anal 1993;7: 209-213.

27. Baynes JW. Role of oxidative stress in development of complications in diabetes. Diabetes 1991;40: 405-412.

28. Ohmori M, Harada K, Kitoh Y, Nagasaka S, Saito T, Fujimura A. The functions of circulatory polymorphonuclear leukocytes in diabetic patients with and without diabetic triopathy. Life Sci 2000;66: 1861-1870. 